\title{
Efectos del maltrato docente en el acoso escolar entre pares*
}

\section{Effects of Teacher's Abuse on Peer School Harassment}

Recepción: 04 Mayo 2017 | Aceptación: 05 Junio 2018

Ana Carolina Reyes Rodríguez

Centro de Investigación en Alimentación y Desarrollo,

México

ORCID: http://orcid.org/0000-0001-8071-1168

Ángel Alberto Valdés Cuervo a

Instituto Tecnológico de Sonora, México

ORCID: http://orcid.org/0000-0001-6559-4151

José Ángel Vera Noriega

Centro de Investigación en Alimentación y Desarrollo,

México

ORCID: http://orcid.org/0000-0003-2764-4431

Carolina Alcántar Nieblas

Instituto Tecnológico de Sonora, México

ORCID: http://orcid.org/0000-0002-1125-6437

a Autor de correspondencia. Correo electrónico: angel.valdes@itson.edu.mx

Para citar este artículo: Reyes Rodríguez, A. C., Valdés Cuervo, A. A., Vera Noriega, J. A., \& Alcántar Nieblas, C. (2018). Efectos del maltrato docente en el acoso escolar entre pares. Universitas Psychologica, 17(5), 1-10. https://doi.org/10.11144/Javeriana.upsy1 7-5.emda

\section{RESUMEN}

En el estudio, se analizan las relaciones entre el maltrato docenteestudiante, clima social de la escuela, apoyo docente y el acoso entre estudiantes. Participaron 1577 estudiantes (800 chicos y 777 chicas), con una edad promedio de 12.94 años $(D E=1.77$ años), pertenecientes a 64 escuelas secundarias públicas de Sonora, México. Se calcula un modelo de ecuaciones estructurales con apoyo del AMOS. De los resultados, se infiere que el maltrato docente favorece de forma directa e indirecta, mediante sus efectos negativos, en el clima escolar y el apoyo docente y la violencia entre pares. Se concluye que el maltrato docente afecta negativamente la dinámica de las escuelas y favorece el acoso escolar. Finalmente, se discuten implicaciones teóricas y prácticas de los hallazgos. Palabras clave

docentes; clima escolar; apoyo social; violencia escolar.

\begin{abstract}
The study analyzes the relationship between teacher-student mistreatment, school climate, teacher's social support and harassment among students. 1,577 students participated on it ( 800 boys and 777 girls), with an average age of 12.94 years $(S D=1.77$ years), they belonged to 64 junior high schools in Sonora, Mexico. A structural equations model is calculated with AMOS support. From the results, it is inferred that teacher mistreatment directly and indirectly favors, through its negative effects on the climate and school support, peer violence. It is concluded that the mistreatment of teachers negatively affects the dynamics of schools and favors school bullying. Finally, the results and their possible theoretical and practical implications of the findings are discussed.

Keywords

teachers; school climate; social support; school violence.
\end{abstract}


El acoso entre estudiantes involucra conductas agresivas intencionales repetidas que se manifiestan en relaciones con marcadas diferencias de poder entre agresores y víctimas (Volk, Dane, \& Marini, 2014). Este problema afecta a escuelas de distintas regiones del mundo (Backhoff \& Pérez-Morán, 2015; Finkelhor, Vanderminden, Turner, Shattuck, \& Hamby, 2016; Garaigordobil \& Machimbarrena, 2017). El acoso perturba la calidad del ambiente del aprendizaje de los estudiantes en las escuelas (Datta, Cornell, \& Huang, 2017; Valdés, Martínez, \& Carlos, 2018). En las víctimas, se reportan depresión, frustración y bajo desempeño académico (Delprato, Akyeampong, \& Dunne, 2017; Lester, Cross, \& Shaw, 2012). Por su parte, en los agresores, se informa escaso compromiso con la escuela, abuso de sustancias y conductas antisociales (Hemphill et al., 2011; Sangalang, Tran, Ayers, \& Marsiglia, 2016).

El acoso entre estudiantes se relaciona con características del contexto escolar (Mucherah, Finch, White, \& Thomas, 2018) y en particular, con la relación docente-estudiante (Casas, Ortega-Ruiz, \& Del Rey, 2015; Longobardi, Iotti, Jungert, \& Settanni, 2018). Los docentes ejercen influencia en el desarrollo académico y psicosocial de los estudiantes, especialmente durante la adolescencia (Quin, Heerde, \& Toumbourou, 2018; Rigby, 2014). En su relación con los docentes, los estudiantes aprenden a experimentar empatía, manejar el estrés y resolver los conflictos pacíficamente con sus pares (Garandeau, Vartio, Poskiparta, \& Salmivalli, 2016; Jungert, Piroddi, \& Thornberg, 2016).

Sin embargo, en ocasiones, la relación docentes-estudiantes favorece el acoso entre pares (Longobardi et al., 2018; Saarento, Karna, Hodges, \& Salmivalli, 2013). La agresión de docentes a estudiantes se asocia con mayor frecuencia de acoso entre pares (Banzon-Librojo, Garabiles, \& Alampay, 2017; Nearchou, 2018). El maltrato implica la percepción del estudiante de ser humillado, denigrado o dañado físicamente por la conducta de los docentes (Benbenishty \& Astor, 2005; Nearchou, 2018). Diversos estudios reportan que entre el 10 y $40 \%$ de los estudiantes son víctimas de maltrato por parte de sus docentes (Chen \& Wei, 2011; Hecker, Goessmann, Nkuba, \& Hermenau, 2018; LucasMolina, Williamson, Pulido, \& Pérez-Albéniz, 2015; Sánchez \& Cepeda, 2014). En México, los escasos estudios del tema confirman también la presencia del problema (Silva \& Corona, 2010; Valdés, Ojeda, Urías, \& Ponce, 2013; Velázquez, 2005).

El maltrato docente-estudiante favorece el acoso entre pares (Banzon-Librojo et al., 2017; Lucas-Molina et al., 2015). Este ocasiona sentimientos negativos en el estudiante (DíazAguado \& Martínez, 2013; Geiger, 2017), legitima la agresión como un recurso para obtener influencia social y resolver conflictos interpersonales (Longobardi et al., 2018; LucasMolina et al., 2015). También afecta la percepción de los alumnos sobre la calidad del clima escolar y el apoyo social de docentes (Lucas-Molina et al., 2015; Van der Zanden, Denessen, \& Scholte, 2015).

El clima escolar se refiere a la calidad de la vida escolar (Cohen, McCabe, Michelli, \& Pickeral, 2009). Un elemento crítico del mismo, asociado a la baja frecuencia de acoso entre pares, es una cercana relación entre docentes y estudiantes (Mucherah et al., 2018; Valdés et al., 2018). La percepción de un trato injusto, escaso afecto y compromiso generan relaciones distantes entre estudiantes y docentes (Fromuth, Davis, Kelly, \& Wakefield, 2015; Gini, Marino, Pozzoli, \& Holt, 2018). Este tipo de relación favorece que el estudiante vivencie escaso apoyo docente para afrontar situaciones de conflictos, en particular el acoso de pares (Sulkowski \& Simmons, 2018; Jungert et al., 2016).

El apoyo escolar involucra la percepción de los estudiantes acerca de las ayudas emocionales, instrumentales e informativas que les brindan los docentes de su escuela (ConnerBurrow, Johnson, Whiteside-Mansell, McKelvey, \& Gargus, 2009). La percepción de falta de apoyo de los docentes favorece la victimización por pares (Díaz \& Bartolomé, 2016; Havik, 2017; Sulkowski \& Simmons, 2018). La carencia de apoyo docente aumenta la vulnerabilidad 
de las víctimas y beneficia las ganancias instrumentales y sociales de la conducta del agresor (Demaray, Malecki, Jenkins, \& Westermann, 2012; Ertesvag, 2016).

Si bien la literatura sugiere que el maltrato docente-estudiante se relaciona con el acoso escolar entre pares, en la investigación realizada se aprecian limitaciones en el estudio de esta temática. En primer lugar, se identifican escasos estudios que indaguen en las relaciones directas e indirectas del maltrato docente-estudiante con el acoso entre estudiantes. En segundo lugar, la investigación acerca de los efectos de la relación docente-estudiante, y en particular del maltrato docente-estudiantes sobre la violencia entre pares es aún incipiente en América Latina y en México.

Por lo tanto, el presente estudio se propone examinar las relaciones entre maltrato docenteestudiante, clima escolar, apoyo social docente y acoso entre estudiantes adolescentes mexicanos. Como se presenta en el siguiente modelo teórico (Figura 1), se espera que el maltrato se relacione, tanto directa como indirectamente, mediante su influencia en el clima escolar y el apoyo social docente, con la mayor frecuencia de acoso entre pares. El presente modelo se considera una aproximación a la comprensión del papel del contexto escolar, especialmente del profesor, para la prevención de la violencia entre pares.

\section{Figura 1}

Modelo hipotético de la relación entre maltrato docente-estudiante, clima escolar, apoyo social docente y acoso entre estudiantes

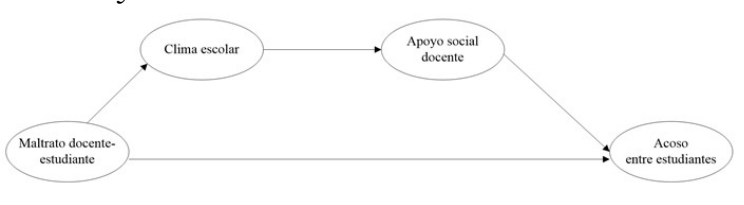

\section{Método}

\section{Participantes}

Los participantes fueron seleccionados de forma no probabilística, con el criterio inicial de que estuvieran representadas las distintas zonas geográficas del estado de Sonora, ubicado en el noroeste de México. Participaron 1577 estudiantes de 64 escuelas secundarias públicas urbanas. Del total de la muestra $800(50.73 \%)$ fueron chicos $(M$ edad $=13.6$ años, $D E=1.3$ años) y $777(49.27 \%)$ chicas ( $M$ edad $=13.4$ años, $D E=1$ años), de los cuales 580 (36.78 \%) cursaban el primer año, 470 (29.81\%) el segundo año y 527 (33.41 \%) el tercer año de secundaria.

\section{Instrumentos}

\section{Maltrato docente-estudiante}

Se adaptó la escala de cinco ítems desarrollada por Mendoza (2006), con la cual se midió la percepción del estudiante acerca de la frecuencia con que fue víctima de maltratos psicológicos o sociales por docentes, durante los últimos dos meses (p. ej. "Me hablan gritando", "Me humillan ante el grupo"). Se utilizó un formato de respuesta tipo Likert con opciones de respuesta desde 0 (nunca) hasta 4 (siempre). Los resultados del análisis factorial confirmatorio muestran que el modelo de medición se ajustó a los datos $\left(\chi^{2}\right.$ $=12.5, g l=8, p=0.13$, SRMR $=0$, AGFI $=0.99, \mathrm{CFI}=0.99, \mathrm{RMSEA}=0.01$, IC 90 $(0$ - 0.03). La fiabilidad de los puntajes medida con el coeficiente Omega de McDonald $(\Omega=$ $0.75)$ y la varianza media extractada (VME $=45$ $\%)$ resultaron aceptables (Zumbo, Gaderman, \& Zeisser, 2007).

\section{Clima escolar}

Se realizó una adaptación de la subescala School Climate Measure Scale ([SCM]; Zullig, Koopman, Patton, \& Ubbes, 2010), que mide con cinco ítems la relación estudiante-docente (p. ej. "Es fácil hablar con los profesores de mi escuela", "Los profesores comprenden mis problemas") que se respondieron en un formato tipo Likert desde 0 (nunca) hasta 4 (siempre). Mediante un análisis factorial confirmatorio se verificó el ajuste del modelo a los datos $\left(\chi^{2}=6.53, g l=3, p=\right.$ 0.088 ; $\mathrm{SRMR}=0.01, \mathrm{AGFI}=0.99, \mathrm{CFI}=0.99$, 
RMSEA $=0.02$, IC $90(0-0.04)$. El valor del coeficiente $\Omega=0.72$ y la VME $=50 \%$ indicaron una adecuada fiabilidad de los puntajes (Zumbo et al., 2007).

\section{Apoyo social docente}

Se utilizó una adaptación de la subescala de la Child and Adolescent Social Support Scale ([CASS]; Malecki \& Demaray, 2002) para medir la percepción de los estudiantes de ayudas que reciben de docentes para enfrentar situaciones de agresión o conflicto con pares. Esta escala constó de cuatro ítems (p. ej. "iAlgún docente te ayuda cuando otro compañero te molesta $y / o$ abusa de ti?"). Se respondió en un formato tipo Likert con opciones de respuesta desde 0 (nunca) hasta 4 (siempre). Los resultados del cálculo de un análisis factorial confirmatorio sugirieron el ajuste del modelo de medida a los datos $\left(\chi^{2}=\right.$ $3.01, g l=3, p=0.082$, SRMR $=0.001$, AGFI $=0.99, \mathrm{CFI}=0.99, \mathrm{RMSEA}=0.02, \mathrm{IC} 90(0$ - 06). El valor de $\Omega=0.84$ y de la $\operatorname{VME}=66$ $\%$ evidenciaron una fiabilidad aceptable de los puntajes (Zumbo et al., 2007).

\section{Victimización por pares}

Se adaptó la escala de autorreporte desarrollada por Valdés y Carlos (2014) para medir la frecuencia de agresiones por pares durante el último mes. La escala se compuso de cinco ítems (p. ej. "Golpeo a compañeros"). Se contestó con un formato tipo Likert con cinco opciones de respuesta: 0 (nunca), 1 (casi nunca, una o dos veces en el mes), 2 ( $a$ veces, tres o cuatro veces en el mes), 3 (casi siempre, cinco a siete veces en el mes) y 4 (siempre, más de siete veces en el mes). En el cálculo del análisis confirmatorio se obtuvieron adecuados índices de ajuste del modelo de medida $\left(\chi^{2}=3.46, g l=4, p=0.483\right.$, SRMR $=0$, AGFI $=0.99, \mathrm{CFI}=0.99$, RMSEA $=0$, IC $(0.00-02)$. Los valores de $\Omega=0.75$ y de la VME $=57 \%$ sugirieron una aceptable fiabilidad de los datos (Zumbo et al., 2007).

\section{Procedimiento}

El estudio contó con la aprobación ética de la Comisión de Investigación del Instituto Tecnológico de Sonora. En primera instancia, se informó a los directivos de las escuelas acerca del objetivo del estudio y se solicitó su autorización para acceder a las instituciones. Posteriormente, se envió a los padres de familia una carta donde se describía el objetivo del estudio y se pidió su consentimiento para que sus hijos participaran en la investigación. Por último, se invitó a los estudiantes a intervenir de forma voluntaria, garantizándoles la confidencialidad de la información.

Los datos perdidos se trataron con el método de imputación por regresión. Con respecto al análisis de datos, para iniciar, se calcularon las medias, desviaciones estándar y correlaciones de Pearson entre todas las variables objeto de estudio. A continuación, se sometió a prueba un modelo estructural de relación entre las variables con apoyo del AMOS. Se utilizó el método de estimación de máxima verosimilitud (ML) con un bootstrap asociado (2 000 repeticiones con un intervalo de confianza del $95 \%$ ), el cual permitió verificar que los resultados de las estimaciones no estuvieran afectados por problemas de normalidad multivariada (Brown, 2015; Kline, 2016).

Se consideraron como índices de ajuste aceptables: $\chi^{2}, p$ (ji-cuadrada y probabilidad asociada) $>0.001$, SRMR (raíz cuadrada de residual estandarizada) $\leq 0.05$, AGFI (índice de bondad de ajuste ajustado), CFI (índice de ajuste comparativo), TLI (índice de Turker-Lewis) $\geq$ 0.95, RMSEA IC 90 (error de la raíz cuadrada de la media de aproximación con su intervalo de confianza) $\leq 0.05$ (Brown, 2015; Kline, 2016) Los efectos indirectos se determinaron mediante el método de bootstrap que realiza el AMOS con un intervalo de confianza del $95 \%$ (Hayes, 2009). 


\section{Resultados}

La Tabla 1 mostró las medias, desviaciones estándar y correlaciones entre las variables. Se encontró una correlación positiva entre el maltrato docente y el acoso entre pares. También el maltrato docente se correlacionó negativamente con el clima escolar y el apoyo social docente. Entre el clima escolar y el apoyo docente se presentó una correlación positiva.

\section{Tabla 1}

Media, desviación estándar y correlación entre las variables involucradas en el estudio

\begin{tabular}{lcccccc}
\hline \multicolumn{1}{c}{ Variables } & $M$ & $D E$ & 1 & 2 & 3 & 4 \\
\hline 1. Acoso & 0.49 & 0.35 & - & & & \\
2. Maltrato docente-estudiante & 0.22 & 0.18 & $0.39 * *$ & - & & \\
3. Clima escolar & 2.99 & 1.16 & $-0.04^{*}$ & $-0.12^{* *}$ & - & \\
4. Apoyo social docente & 3.76 & 1.26 & $-0.12^{* *}$ & $-0.14 * *$ & $0.36^{* *}$ & - \\
\hline \multicolumn{6}{c}{$\mathrm{p}<0.05 * * \mathrm{p}$} & $<0.01$.
\end{tabular}

\section{Ajuste global del modelo estructural}

Tras examinar los resultados de las correlaciones, se decidió incluir todas las variables en el cálculo del modelo de ecuaciones estructurales. Los resultados sugieren un ajuste adecuado de los datos del modelo propuesto $\left(\chi^{2}=97.7, g l=69\right.$, $p=0.01, \mathrm{SRMR}=0.02, \mathrm{AGFI}=0.99, \mathrm{TLI}=$ $0.97, \mathrm{CFI}=0.99$, RMSEA $=0.01$, IC 90 (0.00 0.04). Este modelo explicó el $30 \%$ de la varianza en el acoso entre pares (Figura 2).

\section{Figura 2}

Coeficientes estandarizados y errores estándar (en paréntesis) del análisis de senderos de la relación entre maltrato docente-estudiante, clima escolar, apoyo social docente y acoso entre estudiantes

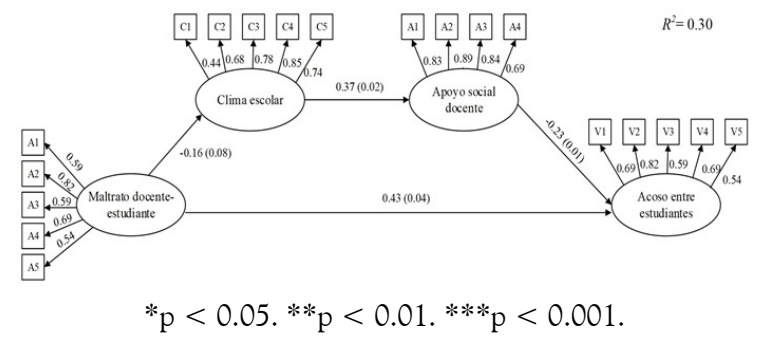

Con respecto a las relaciones directas propuestas en el modelo, los resultados indicaron que el maltrato docente se relacionó en sentido negativo con el clima escolar $(\beta=-0.16, p<$ $0)$ y el apoyo social escolar $(\beta=0.37, p<0)$ y de forma positiva con el acoso entre estudiantes $(\beta=0.43, p<0)$. Así mismo, el clima escolar favoreció el apoyo docente $(\beta=0.37, \mathrm{p}<0)$. Finalmente, se encontró que el apoyo docente disminuye el acoso hacia los estudiantes $(\beta=$ $-0.13, \mathrm{p}<0$ ).

Para determinar los efectos indirectos, se utilizó el método de bootstrap del AMOS con IC 95\% (Hayes, 2009). Se apreció que el maltrato docente $(\beta=0.17$, IC $90(0.17-0.22), p<$ 0.01 ) ejerció un efecto indirecto significativo en el acoso entre pares.

\section{Discusión}

El presente estudio examina las relaciones directas e indirectas del maltrato docenteestudiante con el acoso entre pares, del cual se esperaba que se relacionara de modo directo e indirecto con mayores niveles acoso entre pares. Los resultados muestran que el modelo teórico de relaciones se ajusta a los datos. Además, las hipótesis acerca de las relaciones directas e indirectas son confirmadas por los valores de los coeficientes beta estandarizados.

Con respecto a las relaciones directas de forma consistente con lo reportado en la literatura, los resultados del estudio confirman que el maltrato docente-estudiante favorece la vulnerabilidad de los estudiantes al acoso por pares (Banzon-Librojo et al., 2017; Nearchou, 2018). Los docentes juegan un rol importante en la construcción de la identidad de los adolescentes (Rich \& Schachter, 2012; Uitto, Lutovac, Jokikokko, \& Kaasila, 2018). Al modelar los docentes una conducta de maltrato, los estudiantes aprenden que la agresión es forma aceptable de relacionarse con los otros, lo que favorece la naturalización de la violencia (Banzon-Librojo et al., 2017; Veenstra, Lindenberg, Huitsing, Sainio, \& Salmivalli, 2014). También es factible que el maltrato docente genere una frustración en los estudiantes 
que es canalizada en forma de agresión hacia los pares (Walters \& Espegale, 2018).

Un elemento esencial de un clima escolar positivo es la existencia de relaciones cercanas entre docentes y estudiantes (Valdés et al., 2018). El maltrato erosiona la calidad del clima escolar al provocar relaciones de conflicto con los estudiantes, quienes perciben como injustos y poco confiables a sus docentes (Casas et al., 2015; Lucas-Molina et al., 2015; Longobardi et al., 2018; Van der Zanden et al., 2015).

En relación con las hipótesis indirectas los resultados muestran que el clima escolar y el apoyo docente median los efectos del maltrato en el acoso. El maltrato, al deteriorar el clima escolar, disminuye la percepción del estudiante de apoyo de los docentes para afrontar las agresiones de pares. Esto favorece el acoso, incrementando la vulnerabilidad de las víctimas y empodera a los agresores, quienes no visualizan consecuencias negativas para su comportamiento (Díaz \& Bartolomé, 2016; Ertesvag, 2016; Mucherah et al., 2018).

Desde el punto de vista teórico, el presente estudio amplía la comprensión del rol del docente en el acoso entre estudiantes. Evidencia la necesidad de abordar el acoso escolar desde una perspectiva ecológica-social (Swearer \& Hymel, 2015) que considere, en el análisis de la problemática, las relaciones y características de los contextos sociales donde se desenvuelven los alumnos, y en particular, la dinámica de las relaciones que mantienen con sus docentes.

Desde el punto práctico de los resultados, se infiere que una estrategia eficaz para la prevención del acoso radica en promover en los docentes competencias que les permitan mantener relaciones cercanas con los estudiantes, lo que implica, entre otras cosas, el respeto y el apoyo hacia ellos (Kusché \& Greenberg, 2012; Mucherah et al., 2018). Por lo tanto, es necesario indagar en los factores asociados al maltrato docente para fomentar que los docentes se relacionen de forma no agresiva con los estudiantes.

Así mismo, es necesario enfatizar que, si bien el estudio aporta conocimientos que resultan de provecho para el diseño de programas de prevención de la violencia escolar, los resultados aquí expuestos presentan algunas limitaciones, lo que implica que deben interpretarse con cierta cautela. En primer lugar, el presente estudio es un diseño transversal que no permite establecer relaciones causales entre las variables. Al respecto, se sugiere utilizar en futuras investigaciones diseños experimentales o longitudinales que permitan profundizar en estas relaciones. En segundo lugar, la muestra, aunque resulta suficiente para los análisis estadísticos realizados, es limitada a una región de México, lo que no permite realizar generalizaciones de los hallazgos. En tercer lugar, el maltrato docente se midió desde la percepción de los estudiantes, lo que involucra la necesidad de evaluar el constructo desde otros actores. En cuarto lugar, pueden existir otras variables que influyen en los resultados encontrados que no se consideraron en el presente estudio.

Pese a estas limitaciones, este estudio favorece la comprensión de las relaciones analizadas y resulta relevante para la prevención de la violencia escolar entre pares. En este sentido, los resultados reafirman la importancia del rol del docente en la prevención del acoso escolar y, en particular, de las relaciones que establece con los estudiantes. Asimismo, se concluye que la mejora de las relaciones docente-estudiante se plantea como una línea de intervención prometedora, no solo para disminuir la violencia en los centros educativos, sino también para promover que los estudiantes perciban un clima escolar positivo y el apoyo de los docentes para manejar los conflictos con sus pares.

\section{Referencias}

Backhoff, E., \& Pérez-Morán, J. C. (Coords.). (2015). Segundo estudio internacional sobre la enseñanza y el aprendizaje (TALIS 2013). Resultados de México. México: Instituto Nacional para la Evaluación de la Educación.

Banzon-Librojo, L. A., Garabiles, M. R., \& Alampay, L. P. (2017). Relations between harsh discipline from teacher, perceived 
teacher support, and bullying victimization among high school students. Journal of Adolescence, 57, 18-22. https://doi.org/10.1 016/j.adolescence.2017.03.001

Benbenishty, R., \& Astor, R. A. (2005). School violence in context: Culture, family, school, and gender. Nueva York: Oxford University Press.

Brown, T. A. (2015). Confirmatory factor analysis for applied research (2.a ed.). Nueva York: Guilford.

Casas, J. A., Ortega-Ruiz, R., \& Del Rey, R. (2015). Bullying: The impact of teacher management and trait emotional intelligence. British Journal of Educational Psychology, 85, 407-423. https://doi.org/10. 1111/bjep.12082

Chen, J. K., \& Wei, H. S. (2011). Student victimization by teachers in Taiwan: Prevalence and associations. Child Abuse $\mathcal{E}$ Neglect, 35, 382-390. https://doi.org/10.101 6/j.chiabu.2011.01.009

Cohen, J., McCabe, E. M., Michelli, N. M., \& Pickeral, T. (2009). School climate: Research, policy, practice, and teacher education. Teacher College Record, 111(1), 180-213. Recuperado de http://www.ijvs.or g/files/Publications/School-Climate.pdf

Conner-Burrow, N. A., Johnson, D. L., Whiteside-Mansell, L., McKelvey, L., \& Gargus, R. A. (2009). Adults matter: Protecting children from the negative impacts of bullying. Psychology in the Schools, 46, 593-604. https://doi.org/10.1002/pits.2 0400

Datta, P., Cornell, D., \& Huang, F. (2017). The toxicity of bullying by teacher and other school staff. School Psychology Review, 46, 335-348. https://doi.org/10.17105/SPR2017-0001.V46-4

Delprato, M., Akyeampong, K., \& Dunne, M. (2017). The impact of bullying on students' learning in Latin America: A matching approach for 15 countries. International Journal of Educational Development, 52, 37-57. https://doi.org/10.1016/j.ijedudev.2 016.10 .002
Demaray, M. K., Malecki, C. K., Jenkins, L., \& Westermann, L. (2012). Social support in the lives of students involved in aggressive and bullying behaviors. En S. Jimerson, A. Nickerson, M. Mayer \& M. Furlong (Eds.), Handbook of school violence and school safety. International research and practice (2.a ed., pp. 57-67). Nueva York: Routledge.

Díaz, E., \& Bartolomé, R. (2016). Social support as a school victimisation risk factor. Journal of Child and Family Studies, 25, 3473-3480. h ttps://doi.org/10.1007/s10826-016-0503-9

Díaz-Aguado, M. J., \& Martínez, R. (2013). Peer bullying and disruptioncoercion escalations in student-teacher relationships. Psicothema, 25, 206-213. http s://doi.org/10.7334/psicothema2012.312

Ertesvag, S. K. (2016). Students who bully and their perceptions of teacher support and monitoring. British Educational Research Journal, 42, 826-850. https://doi.org/10.100 2/berj.3240

Finkelhor, D., Vanderminden, J., Turner, H., Shattuck, A., \& Hamby, S. (2016). Atschool victimization and violence exposure assessed in a National Household Survey of children and youth. Journal of School Violence, 15, 67-90. https://doi.org/10.1080 /15388220.2014.952816

Fromuth, M. E., Davis, T. L., Kelly, D. B., \& Wakefield, C. (2015). Descriptive features of student psychological maltreatment by teacher. Journal of Children Adolescent Trauma, 8, 127-135. https://doi.org/1007/s4 0653-015-0042-3

Garaigordobil, M., \& Machimbarrena, J. M. (2017). Stress, competence, and parental educational styles in victims and aggressors of bullying and cyberbullying. Psicothema, 29, 335-340. https://doi.org/10.7334/psicot hema2016.258

Garandeau, C. F., Vartio, A., Poskiparta, E., \& Salmivalli, C. (2016). School bullies' intention to change behavior following teacher interventions: Effects of the empathy arousal, condemning of bullying, and blaming of the perpetrator. Prevention 
Science, 17, 1034-1043. https://doi.org/10.1 007/s11121-016-0712-x

Geiger, B. (2017). Sixth grades in Israel recount their experience of verbal abuse by teachers in the classroom. Child Abuse $\mathbb{E}$ Neglect, 63, 95-105. https://doi.org/10.1016/j.chiabu.20 16.11.019

Gini, G., Marino, C., Pozzoli, T., \& Holt, M. (2018). Associations between peer victimization, perceived teacher unfairness, and adolescents' adjustment and wellbeing. Journal of School Psychology, 67, 56-68. https://doi.org/10.1016/j.jsp.2017.0 9.005

Havik, T. (2007). Bullying victims' perceptions of classroom interaction, school effectiveness and school improvement. School Effectiveness and School Improvement, 28, 826-850. https://doi.org/10.1080/09243453 .2017 .1294609

Hayes, A. F. (2009). Beyond Baron and Kenny: Statistical mediation analysis in the new millennium. Communication Monographs, 76, 408-420. https://doi.org/10.1080/03637 750903310360

Hecker, T., Goessmann, K., Nkuba, M., \& Hermenau, K. (2018). Teachers' stress intensifies violent disciplining in Tanzanian secondary schools. Child Abuse $\mathbb{E}$ Neglect, 76, 173-183. https://doi.org/10.1016/j.chia bu.2017.10.019

Hemphill, S. A., Kotevski, A., Herrenkohl, T. I., Bond, L., Kim, M. J., Toumbourou, J. W., \& Catalano, R. F. (2011). Longitudinal consequences of adolescent bullying perpetration and victimization: A study of students in Victoria, Australia. Criminal Behaviour and Mental Health, 21, 107-116. https://doi.org/10.1002/cbm.802

Jungert, T., Piroddi, B., \& Thornberg, R. (2016). Early adolescents' motivations to defend victims in school bullying and their perceptions of student-teacher relationships: A self-determination theory approach. Journal of Adolescence, 53, 75-90. https://doi.org/10.1016/j.adolescenc e.2016.09.001
Kline, R. B. (2016). Principles and practice of structural equation modeling (4.a ed.). Nueva York: Guilford.

Kusché, C., \& Greenberg, M. (2012). The PATHS curriculum: Promoting emotional literacy, prosocial behavior, and caring classroom. En S. Jimerson, A. Nickerson, M. Mayer \& M. Furlong (Eds.), Handbook of school violence and safety (pp. 435-446). Nueva York: Routledge.

Lester, L., Cross, D., \& Shaw, T. (2012). Problem behaviors, traditional bullying and cyberbullying among adolescents: Longitudinal analyses. Emotional and Behavioural Difficulties, 17, 435-447. https:/ /doi.org/10.1080/13632752.2012.704313

Longobardi, C., Iotti, N. O., Jungert, T., \& Settanni, M. (2018). Student-teacher relationships and bullying: The role of student social status. Journal of Adolescence, 63, 1-10. https://doi.org/0.1016/j.adolescen ce.2017.12.001

Lucas-Molina, B., Williamson, A. A., Pulido, R., \& Pérez-Albéniz, A. (2015). Effects of teacher-student relationships of peer harassment: A multilevel study. Psychology in the School, 52, 298-315. https://doi.org/1 $0.1002 /$ pits. 21.822

Malecki, C. K., \& Demaray, M. K. (2002). Measuring perceived social support: Development of the Child and Adolescent Social Support Scale (CASS). Psychology in the School, 39, 1-18. https://doi.org/10.1002 /pits.10004

Mendoza, B. (2006). Las dos caras de la violencia escolar. El maltrato en la interacción profesoralumno y entre iguales (Tesis doctoral inédita). Universidad Complutense de Madrid, Madrid.

Mucherah, W., Finch, H., White, T., \& Thomas, K. (2018). The relationship of school climate, teacher defending and friends on students' perception of bullying in high school. Journal of Adolescence, 62, 128-139. https://doi.org/10.1016/j.adolesce nce.2017.11.012

Nearchou, F. (2018). Resilience following emotional abuse by teachers: Insights from 
a cross-sectional study with Greek students. Child Abuse $\mathfrak{E}$ Neglect, 78, 96-106. https:// doi.org/10.1016/j.chiabu.2017.10.012

Quin, D., Heerde, J. A., \& Toumbourou, J. W. (2018). Teacher support within and ecological model of adolescent development: Predictors of school engagement. Journal of School Psychology, 69, 1-15. https://doi.org/10.1016/j.jsp.2018 .04 .003

Rich, Y., \& Schachter, E. P. (2012). High school identity climate and student identity development. Contemporary Educational Psychology, 37, 218-228. https://doi.org/10. 1016/j.cedpsych.2011.06.002

Rigby, K. (2014). How teacher address cases of bullying in schools: A comparison of five reactive approaches. Educational Psychology in Practice, 30, 409-419. https://doi.org/10. 1080/02667363.2014.949629

Saarento, S., Karna, A., Hodges, E. V., \& Salmivalli, C. (2013). Student-, classroom-, and school-level risk factors for victimization. Journal of School Psychology, 51, 421-434. https://doi.org/10.1016/j.jsp.2 013.02 .002

Sánchez, E., \& Cepeda, E. (2014). ¿Los estudiantes de educación básica y media son víctimas de maltrato por parte de sus profesores? Un estudio de percepción. Psicología desde el Caribe, 31(2), 223-242. Recuperado de http://rcientificas.uninorte.edu.co/index .php/psicologia/article/view/5214

Sangalang, C. C., Tran, A. G. T. T., Ayers, S. L., \& Marsiglia, F. F. (2016). Bullying among urban Mexican-heritage youth: Exploring risk for substance use by status as a bully, victim, and bully-victim. Children and Youth Services Review, 61, 216-221. https://doi.org /10.1016/j.childyouth.2015.12.019

Silva, J. L., \& Corona, A. (2010). Violencia en las escuelas del Distrito Federal. La experiencia de la Unidad para la Atención al Maltrato y Abuso Sexual, 2001-2007. Revista Mexicana de Investigación Educativa, 15(46), 735-770. Recuperado de http://www.redalyc.org/pdf/ 140/14015585004.pdf
Sulkowski, M., \& Simmons, J. (2018). The protective role of teacher-student relationships against peer victimization and psychosocial distress. Psychology in the School, 55, 137-150. https://doi.org/10.100 2/pits. 22086

Swearer, S. M., \& Hymel, S. (2015). Understanding the psychology of bullying. American Psychologist, 70, 344-353. https:// doi.org/10.1037/a0038929

Uito, M., Lutovac, S., Jokikokko, K., \& Kaasila, R. (2018). Recalling life changing teachers: Positive memories of teacher-student relationships and the emotions involved. International Journal of Educational Research, 87, 47-56. https://doi.org/10.1016/j.ijer.201 7.11 .004

Valdés, A. A., \& Carlos, E. A. (2014). Relación entre el autoconcepto, el clima familiar y el clima escolar con el bullying en estudiantes de secundaria. Avances en Psicología Latinoamericana, 32, 447-457. htt ps://doi.org/10.12804/apl32.03.201407

Valdés, A. A., Martínez, B., \& Carlos, E. C. (2018). The role of teaching practices in the prevention of school violence among peers. Revista de Psicodidáctica, 22, 33-38. https:// doi.org/10.1016/j.psicod.2017.05.006

Valdés, A. A., Ojeda, A., Urías, M., \& Ponce, D. (2013). Factores de riesgo en estudiantes de secundaria con conductas violentas hacia los compañeros. En J. Vales, A. A. Valdés, J. Angulo, C. García \& I. González (Eds.), Investigación psicoeducativa en Sonora. Resultados y propuestas de acción (pp. 1- 19). México: Pearson.

Van den Zanden, P. J., Denessen, E. J., \& Scholte, R. H. (2015). The effects of general and bullying-specific teacher behaviors on pupils' bullying behaviors at school. School Psychology International, 36, 467-485. https: //doi.org/10.1177/0143034315592754

Veenstra, R., Lindenberg, S., Huitsing, G., Sainio, M., \& Salmivalli, C. (2014). The role of teachers in bullying: The relation between antibullying attitudes, efficacy, and efforts to reduce bullying. Journal of Educational 
Psychology, 106, 1135-1143. https://doi.org/ $10.1037 / \mathrm{a} 0036110$

Velázquez, L. (2005). Experiencias estudiantiles con la violencia en la escuela. Revista Mexicana de Investigación Educativa, 10(26), 739-764. Recuperado de http://www.redaly c.org/pdf/140/14002607.pdf

Volk, A. A., Dane, A. V., \& Marini, Z. A. (2014). What is bullying? A theoretical redefinition. Developmental Review, 34, 327-343. https://doi.org/10.1016/j.dr.2014. 09.001

Walters, G. D., \& Espegale, D. L. (2018). From victim to victimizer: Hostility, anger, and depression as mediators of the bullying victimization-bullying perpetration association. Journal of School Psychology, 68, 73-83. https://doi.org/10.1016/j.jsp.201 7.12 .003

Zullig, K. J., Koopman, T. M., Patton, J. M., \& Ubbes, V. A. (2010). School climates: historical review, instrument development, and school assessment. Journal of Psychoeducational Assessment, 28, 139-152. https://doi.org/10.1177/07342829 09344205

Zumbo, B. D., Gaderman, A. M., \& Zeisser, C. (2007). Ordinal version of coefficients Alpha and Theta for Likert rating scales. Journal of Modern Applied Statistical Methods, 6(1), 21-29. https://doi.org/10.22 237/jmasm/1177992180

\section{Notas}

* Artículo de investigación. Financiado por el Programa de Fortalecimiento y Apoyo a la Investigación (PROFAPI), Instituto Tecnológico de Sonora. 